\title{
RADIATION EFFECT ON THREE DIMENSIONAL VERTICAL CHANNEL FLOW THROUGH POROUS MEDIUM
}

\author{
M. GURIA \\ Department of Mathematics \\ Ghatal Rabindra Satabarsiki Mahavidyalaya \\ Paschim Midnapore, West Bengal, INDIA \\ E-mail: mrinmoy@yahoo.com
}

\begin{abstract}
The flow of a viscous incompressible fluid through a vertical channel in the presence of radiation immersed in a porous medium has been studied. Approximate solutions have been obtained for the velocity and temperature fields, shear stresses and rate of heat transfer using the perturbation technique. It is found that the primary velocity decreases with an increase in the radiation parameter as well as the Prandtl number for cooling of the plate. It is also found that with an increase in the permeability parameter, the primary velocity increases for cooling of the plate. The magnitude of the secondary velocity decreases near the plate $y=0$ and increases near the plate $y=d$ with an increase in the permeability parameter. The temperature distribution decreases with an increase of the radiation parameter as wall as the Prandtl number for cooling of the plate. The shear stresses and the rate of heat transfer, which are of physical interest, are presented in the form of tables.
\end{abstract}

Kew words: three-dimensional, injection, periodic suction, permeability, porous medium.

\section{Introduction}

Free convective flows with periodic permeability through porous media play an important role in chemical engineering for filtration and purification processes, turbo-machinery and in aerospace technology. Such flows include several practical applications, for example, geothermal reservoirs, drying of porous solids, thermal insulation, cooling of nuclear reactors and underground energy transport. Rapits (1983) investigated the problem of an unsteady flow through a porous medium bounded by an infinite porous plate subjected to a constant suction and variable temperature. Rapits and Perdikis (1985) also investigated the free convective flow through a porous medium bounded by a vertical porous plate with constant suction. Varshney (1979) discussed the oscillatory two dimensional flow through a porous medium bounded by a porous plate. Singh et al. (1988) studied the three dimensional convective flow through a porous medium bounded by an infinite vertical porous plate with periodic suction. Ahmed and Ahmed (2004) analysed the effect of a two dimensional MHD oscillatory flow along a uniformly moving infinite vertical porous plate bounded by a porous medium. However, these studied are confined to normal temperature of the surrounding medium. If the temperature of the surrounding fluid is rather high, radiation effects play an important role and this situation does exist in space technology. Nuclear power plants, gas turbines and the various propulsion devices for aircraft, missiles, satellites are examples of such engineering areas. In this case one has to take into consideration the effects of radiation and free convection. Seddeek (2000) investigated the effect of radiation past a moving plate with variable viscosity. The effect of radiation and magnetic field on the flow past a vertical plate was discussed by Takhar et al. (1996). Rapits (1998) also studied the effect of radiation and free convection on a steady flow past a vertical porous plate through a porous medium. Sharma et al. (2007) studied the effect of radiation in a three dimensional Couette flow subjected to a periodic suction velocity distribution. Recently, Guria et al. (2010) investigated the effect of radiation on a three dimensional flow in a vertical channel subjected to a periodic suction. Guria et al. (2011) also investigated the effect of radiation on three dimensional flow past a vertical porous plate in the presence of transverse magnetic field. Pathak et al. (2006) 
studied the radiation effects on an unsteady free convective flow through a porous medium bounded by an oscillating plate with variable wall temperature. The effect of radiation on a three dimensional flow past a vertical channel through a porous medium has not been studied. Our aim is to study the effect of radiation and permeability of the medium on a three -dimensional flow of fluid through a vertical channel.

\section{Basic equations}

Consider the steady flow of a viscous, incompressible fluid between vertical parallel porous plates separated by a distance $d$. Here the $x^{\star}$ - axis is chosen along the direction of the flow, $y^{\star}-$ axis is perpendicular to the wall of the channel and $z^{\star}$ - axis normal to the $x^{\star} y^{\star}$-plane [see Fig.1]. The temperature at the plates $y^{*}=0$ and $y^{*}=d$ are $T_{w}$ and $T_{0}\left(T_{w}>T_{0}\right)$, respectively.

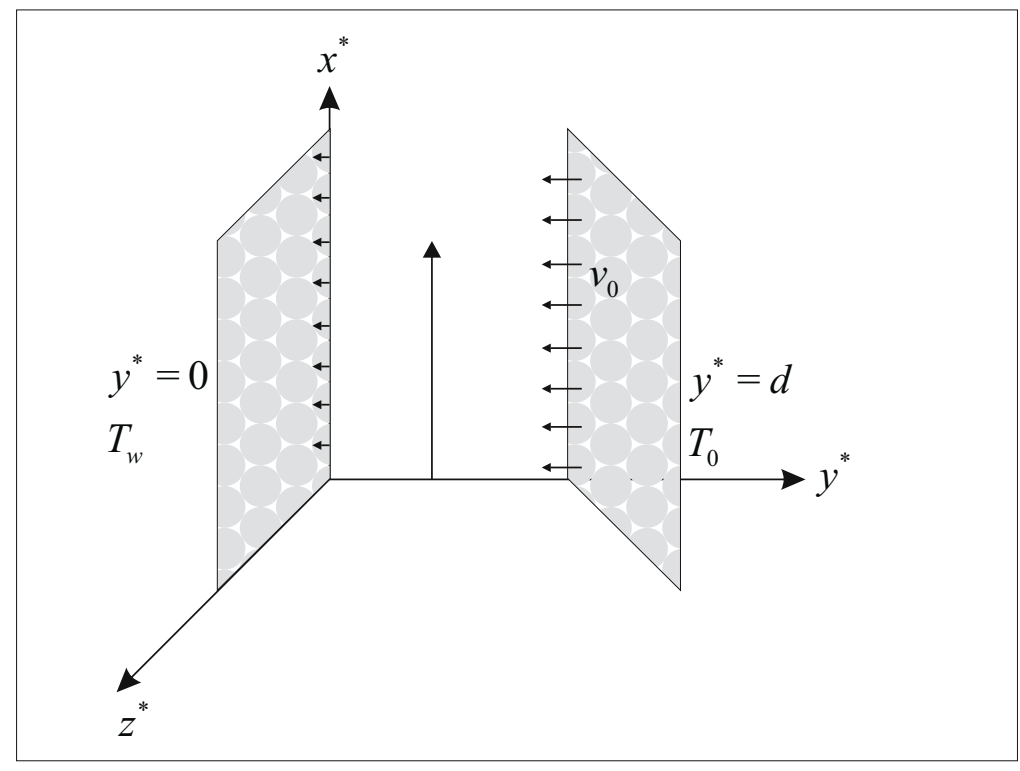

Fig.1. Physical model and co-ordinate system.

The plate $y^{\star}=d$ is subjected to a uniform injection $V_{0}$ and the plate $y^{*}=0$ to a periodic suction velocity distribution of the form

$$
v^{*}=-V_{0}\left[1+\varepsilon \cos \left(\frac{\pi z^{*}}{d}\right)\right]
$$

where $\varepsilon(\ll 1)$ is the amplitude of the suction velocity.

The velocity and temperature fields are independent of $x$ since the channel is infinite long along the $x$-direction. The flow itself will be three dimensional due to cross flow.

Let $u^{*}, v^{*}, w^{*}$ be the velocity components in the directions of $x^{*}-, y^{*}-, z^{*}-$ axes respectively. The problem is governed by the following equations

$$
\frac{\partial v^{*}}{\partial y^{*}}+\frac{\partial w^{*}}{\partial z^{*}}=0
$$




$$
\begin{aligned}
& v^{*} \frac{\partial u^{*}}{\partial y^{*}}+w^{*} \frac{\partial u^{*}}{\partial z^{*}}=v\left(\frac{\partial^{2} u^{*}}{\partial y^{* 2}}+\frac{\partial^{2} u^{*}}{\partial z^{* 2}}\right)+g \beta\left(T^{*}-T_{0}\right)-\frac{v u^{*}}{K^{*}}, \\
& v^{*} \frac{\partial v^{*}}{\partial y^{*}}+w^{*} \frac{\partial v^{*}}{\partial z^{*}}=-\frac{1}{\rho} \frac{\partial p^{*}}{\partial y^{*}}+v\left(\frac{\partial^{2} v^{*}}{\partial y^{* 2}}+\frac{\partial^{2} v^{*}}{\partial z^{* 2}}\right)-\frac{v v^{*}}{K^{*}}, \\
& v^{*} \frac{\partial w^{*}}{\partial y^{*}}+w^{*} \frac{\partial w^{*}}{\partial z^{*}}=-\frac{1}{\rho} \frac{\partial p^{*}}{\partial z^{*}}+v\left(\frac{\partial^{2} w^{*}}{\partial y^{* 2}}+\frac{\partial^{2} w^{*}}{\partial z^{* 2}}\right)-\frac{v w^{*}}{K^{*}}, \\
& v^{*} \frac{\partial T^{*}}{\partial y^{*}}+w^{*} \frac{\partial T^{*}}{\partial z^{*}}=v\left(\frac{\partial^{2} T^{*}}{\partial y^{* 2}}+\frac{\partial^{2} T^{*}}{\partial z^{* 2}}\right)-\frac{1}{\rho C_{p}} \frac{\partial q_{r}^{*}}{\partial y^{*}}
\end{aligned}
$$

where $v$ is the kinematic coefficient of viscosity, $\rho$ is the density, $p^{*}$ is the fluid pressure, $g$ is the acceleration due to gravity, $\beta$ is the thermal expansion and $C_{p}$ is the specific heat at constant pressure. $K^{*}$ is the permeability of the medium.

The equation of conservation of radiative heat transfer per unit volume for all wavelength is

$$
\nabla \cdot q_{r}^{*}=\int_{0}^{\infty} K_{\lambda}\left(T^{*}\right)\left(4 e_{\lambda h}\left(T^{*}\right)-G_{\lambda}\right) d \lambda
$$

where $e_{\lambda h}$ is Plank's function and the incident radiation $G_{\lambda}$ is defined as

$$
G_{\lambda}=\frac{1}{\pi} \int_{\Omega=4 \pi} e_{\lambda}(\Omega) d \Omega
$$

$\nabla . q_{r}^{*}$ is the radiative flux divergence and $\Omega$ is the solid angle. Now, for an optically thin fluid exchanging radiation with an isothermal flat plate at temperature $T_{0}$ and according to the above definition for the radiative flux divergence and Kirchhoffs law, the incident radiation is given by $G_{\lambda}=4 e_{\lambda h}\left(T_{0}\right)$ then

$$
\nabla \cdot q_{r}^{*}=4 \int_{0}^{\infty} K_{\lambda}\left(T^{*}\right)\left(e_{\lambda h}\left(T^{*}\right)-e_{\lambda h}\left(T_{0}\right)\right) d \lambda
$$

Expanding $K_{\lambda}\left(T^{*}\right)$ and $e_{\lambda h}\left(T_{0}\right)$ in a Taylor series around $T_{0}$, for small $\left(T^{*}-T_{0}\right)$, we can rewrite the radiative flux divergence as

$$
\nabla \cdot q_{r}^{*}=4\left(T^{*}-T_{0}\right) \int_{0}^{\infty} K_{\lambda_{0}}\left(\frac{\partial e_{\lambda h}}{\partial T}\right)_{0} d \lambda
$$

where $K_{\lambda_{0}}=K_{\lambda\left(T_{0}\right)}$.

Hence an optical thin limit for a non-gray gas near equilibrium, the following relation holds 


$$
\nabla \cdot q_{r}^{*}=4\left(T^{*}-T_{0}\right) I,
$$

and hence

$$
\frac{\partial q_{r}^{*}}{\partial y^{*}}=4\left(T^{*}-T_{0}\right) I
$$

where

$$
I=\int_{0}^{\infty} K_{\lambda_{0}}\left(\frac{\partial e_{\lambda h}}{\partial T}\right)_{0} d \lambda
$$

The boundary conditions of the problem are

$$
\begin{aligned}
& u^{*}=0, \quad v^{*}=-V_{0}\left[1+\varepsilon \cos \left(\frac{\pi}{d} z^{*}\right)\right], \quad w^{*}=0, \quad T^{*}=T_{w} \quad \text { at } \quad y^{*}=0, \\
& u^{*}=0, \quad v^{*}=-V_{0}, \quad w^{*}=0, \quad T^{*}=T_{0}, \quad p^{*}=p_{\infty} \quad \text { at } \quad y^{*}=d .
\end{aligned}
$$

Introducing the non dimensional variables

$$
y=\frac{y^{*}}{d}, \quad z=\frac{z^{*}}{d}, \quad p=\frac{p^{*}}{\rho V_{0}^{2}}, \quad u=\frac{u^{*}}{V_{0}}, \quad v=\frac{v^{*}}{V_{0}}, \quad w=\frac{w^{*}}{V_{0}}, \quad \theta=\frac{\left(T^{*}-T_{0}\right)}{\left(T_{w}-T_{0}\right)} .
$$

Equations (2.2)-(2.6) become

$$
\begin{aligned}
& \frac{\partial v}{\partial y}+\frac{\partial w}{\partial z}=0 \\
& v \frac{\partial u}{\partial y}+w \frac{\partial u}{\partial z}=\frac{1}{\operatorname{Re}}\left(\frac{\partial^{2} u}{\partial y^{2}}+\frac{\partial^{2} u}{\partial z^{2}}\right)+\operatorname{Gr} \theta-\frac{u}{K}, \\
& v \frac{\partial v}{\partial y}+w \frac{\partial v}{\partial z}=-\frac{\partial p}{\partial y}+\frac{1}{\operatorname{Re}}\left(\frac{\partial^{2} v}{\partial y^{2}}+\frac{\partial^{2} v}{\partial z^{2}}\right)-\frac{v}{K}, \\
& v \frac{\partial w}{\partial y}+w \frac{\partial w}{\partial z}=-\frac{\partial p}{\partial z}+\frac{1}{\operatorname{Re}}\left(\frac{\partial^{2} w}{\partial y^{2}}+\frac{\partial^{2} w}{\partial z^{2}}\right)-\frac{w}{K}, \\
& v \frac{\partial \theta}{\partial y}+w \frac{\partial \theta}{\partial z}=\frac{1}{\operatorname{RePr}}\left(\frac{\partial^{2} \theta}{\partial y^{2}}+\frac{\partial^{2} \theta}{\partial z^{2}}\right)-F \theta
\end{aligned}
$$


where $\operatorname{Re}=V_{0} d / \nu$, the Reynolds number, $\operatorname{Pr}=v / \rho$, the Prandtl number and $\operatorname{Gr}=d g \beta\left(T_{w}-T_{0}\right) / V_{0}^{2}$, the Grashof number, $F=4 I d / \rho C_{p} V_{0}$, the radiation parameter. Using Eqs (2.8), the boundary conditions (2.7) become

$$
\begin{aligned}
& u=0, \quad v=-[1+\varepsilon \cos (\pi z)], \quad w=0, \quad \theta=1 \quad \text { at } \quad y=0, \\
& u=0, \quad v=-1, \quad w=0, \quad \theta=0, \quad p=\frac{p_{\infty}}{\rho V^{2}} \quad \text { at } \quad y=1 .
\end{aligned}
$$

\section{Solution of the problem}

In order to solve the differential Eqs (2.9)-(2.13), we assume the solution of the following form

$$
\begin{aligned}
& u(y, z)=u_{0}(y)+\varepsilon u_{l}(y, z)+\varepsilon^{2} u_{2}(y, z)+\cdots, \\
& v(y, z)=v_{0}(y)+\varepsilon v_{1}(y, z)+\varepsilon^{2} v_{2}(y, z)+\cdots, \\
& w(y, z)=w_{0}(y)+\varepsilon w_{l}(y, z)+\varepsilon^{2} w_{2}(y, z)+\cdots \\
& p(y, z)=p_{0}(y)+\varepsilon p_{l}(y, z)+\varepsilon^{2} p_{2}(y, z)+\cdots \\
& \theta(y, z)=\theta_{0}(y)+\varepsilon \theta_{l}(y, z)+\varepsilon^{2} \theta_{2}(y, z)+\cdots
\end{aligned}
$$

On substituting (3.1) in Eqs (2.9)-(2.13) and equating the terms independent of $\varepsilon$, we get the following system of differential equations

$$
\begin{aligned}
& v_{0}^{\prime}=0, \\
& u_{0}^{\prime \prime}-\operatorname{Re} v_{0} u_{0}^{\prime}-\frac{\operatorname{Re} u_{0}}{K}=-\operatorname{ReGr} \theta_{0}, \\
& \theta_{0}^{\prime \prime}-\operatorname{RePr} v_{0} \theta_{0}^{\prime}-F \operatorname{RePr} \theta_{0}=0
\end{aligned}
$$

where primes denote differentiation with respect to $y$ and the corresponding boundary conditions become

$$
u_{0}=0, \quad v_{0}=-1, \quad \theta_{0}=1 \quad \text { at } \quad y=0 \quad \text { and } \quad u_{0}=0, \quad v_{0}=-1, \quad \theta_{0}=0 \quad \text { at } y=1
$$

The solutions of Eqs (3.2) to (3.4), subject to the boundary conditions (3.5) are

$$
v_{0}(y)=-1
$$




$$
\begin{aligned}
& \theta_{0}(y)=\frac{1}{\left(e^{-m_{2}}-e^{-m_{1}}\right)}\left[e^{-m_{2}} e^{-m_{1} y}-e^{-m_{1}} e^{-m_{2} y}\right], \\
& u_{0}(y)=\sum_{i=0}^{4} A_{i} e^{-m_{i} y}
\end{aligned}
$$

where

$$
\begin{aligned}
& m_{l}=\frac{1}{2}\left\{\operatorname{RePr}+\sqrt{\operatorname{Re}^{2} \operatorname{Pr}^{2}+4 F \operatorname{RePr}}\right\}, \\
& m_{2}=\frac{1}{2}\left\{\operatorname{RePr}-\sqrt{\operatorname{Re}^{2} \operatorname{Pr}^{2}+4 F \operatorname{RePr}}\right\}, \\
& m_{3}=\frac{1}{2}\left\{\operatorname{Re}+\sqrt{\operatorname{Re}^{2}+\frac{4 \mathrm{Re}}{K}}\right\}, \\
& m_{4}=\frac{1}{2}\left\{\operatorname{Re}-\sqrt{\operatorname{Re}^{2}+\frac{4 \mathrm{Re}}{K}}\right\}, \\
& A_{l}=\frac{-\operatorname{Gr} \operatorname{Re} e^{-m_{2}}}{\left(e^{-m_{2}}-e^{-m_{l}}\right)\left(m_{l}^{2}-m_{l} \operatorname{Re}-\operatorname{Re} / K\right)}, \\
& A_{2}=\frac{\operatorname{Gr} \operatorname{Re} e^{-m_{l}}}{\left(e^{-m_{2}}-e^{-m_{l}}\right)\left(m_{2}^{2}-m_{2} \operatorname{Re}-\operatorname{Re} / K\right)}, \\
& A_{3}=\frac{-1}{\left(e^{-m_{3}}-e^{m_{4}}\right)}\left[A_{1}\left(e^{-m_{1}}-e^{-m_{4}}\right)+A_{2}\left(e^{-m_{2}}-e^{-m_{4}}\right)\right], \\
& A_{4}=\frac{1}{\left(e^{-m_{3}}-e^{-m_{4}}\right)}\left[A_{1}\left(e^{-m_{1}}-e^{-m_{3}}\right)+A_{4}\left(e^{-m_{2}}-e^{-m_{3}}\right)\right] .
\end{aligned}
$$

On substituting (3.1) in Eqs (2.9)-(2.13) and equating the coefficient of $\varepsilon$, we get the following system of differential equations

$$
\begin{aligned}
& \frac{\partial v_{1}}{\partial y}+\frac{\partial w_{1}}{\partial z}=0 \\
& v_{0} \frac{\partial u_{1}}{\partial y}+v_{1} \frac{\partial u_{0}}{\partial y}=\frac{1}{\operatorname{Re}}\left(\frac{\partial^{2} u_{1}}{\partial y^{2}}+\frac{\partial^{2} u_{1}}{\partial z^{2}}\right)+\operatorname{Gr} \theta_{1}-\frac{u_{1}}{K},
\end{aligned}
$$




$$
\begin{aligned}
& v_{0} \frac{\partial v_{1}}{\partial y}=-\frac{\partial p_{1}}{\partial y}+\frac{1}{\operatorname{Re}}\left(\frac{\partial^{2} v_{1}}{\partial y^{2}}+\frac{\partial^{2} v_{1}}{\partial z^{2}}\right)-\frac{v_{1}}{K}, \\
& v_{0} \frac{\partial w_{1}}{\partial y}=-\frac{\partial p_{1}}{\partial z}+\frac{1}{\operatorname{Re}}\left(\frac{\partial^{2} w_{1}}{\partial y^{2}}+\frac{\partial^{2} w_{1}}{\partial z^{2}}\right)-\frac{w_{1}}{K}, \\
& v_{0} \frac{\partial \theta_{1}}{\partial y}+v_{1} \frac{\partial \theta_{0}}{\partial y}=\frac{1}{\operatorname{RePr}}\left(\frac{\partial^{2} \theta_{1}}{\partial y^{2}}+\frac{\partial^{2} \theta_{1}}{\partial z^{2}}\right)-F \theta_{1} .
\end{aligned}
$$

The corresponding boundary conditions become

$$
\begin{aligned}
& u_{1}=0, \quad v_{1}=-\cos (\pi z), \quad w_{1}=0, \quad \theta_{1}=0 \quad \text { at } \quad y=0, \\
& u_{1}=0, \quad v_{1}=0, \quad w_{1}=0, \quad \theta_{1}=0 \quad \text { at } \quad y=1 .
\end{aligned}
$$

These are the linear partial differential equations describing the three dimensional flow. To solve Eqs (3.10)-(3.14), we assume velocity components and pressure in the following form

$$
\begin{aligned}
& u_{1}(y, z)=u_{11}(y) \cos (\pi z), \\
& v_{1}(y, z)=v_{11}(y) \cos (\pi z), \\
& w_{1}(y, z)=-\frac{1}{\pi} v_{11}^{\prime}(y) \sin (\pi z), \\
& p_{1}(y, z)=p_{11}(y) \cos (\pi z), \\
& \theta_{1}(y, z)=\theta_{11}(y) \cos (\pi z)
\end{aligned}
$$

$v_{l}$ and $w_{l}$ are so chosen that the continuity Eq.(3.10) is satisfied automatically.

Substituting (3.16) in Eqs (3.10)-(3.14) and comparing the coefficients of harmonic terms, we obtain the following set of differential equations

$$
\begin{aligned}
& v_{11}^{\prime \prime}+\operatorname{Re} v_{11}^{\prime}-\left(\pi^{2}+\frac{\operatorname{Re}}{K}\right) v_{11}=\operatorname{Re} p_{11}^{\prime}, \\
& v_{11}^{\prime \prime \prime}+\operatorname{Re} v_{11}^{\prime \prime}-\left(\pi^{2}+\frac{\operatorname{Re}}{K}\right) v_{11}^{\prime}=\operatorname{Re} \pi^{2} p_{11}, \\
& \theta_{11}^{\prime \prime}+\operatorname{RePr} \theta_{11}^{\prime}-\left(F \operatorname{RePr}+\pi^{2}\right) \theta_{11}=\operatorname{RePr} v_{11} \theta_{0}^{\prime},
\end{aligned}
$$




$$
u_{11}^{\prime \prime}+\operatorname{Re} u_{11}^{\prime}-\left(\pi^{2}+\frac{\operatorname{Re}}{K}\right) u_{11}=\operatorname{Re} v_{11} u_{0}^{\prime}-\operatorname{GrRe} \theta_{11}
$$

When $K \rightarrow \infty$ Eqs (3.17)-(3.20) coincide with Eqs (3.17)-(3.20) of Guria and Jana (2010). The corresponding boundary conditions are

$$
\begin{array}{ll}
u_{11}=0, \quad v_{11}=-1, \quad v_{11}^{\prime}=0, \quad \theta_{11}=0 \quad \text { at } & y=0, \\
u_{11}=0, \quad v_{11}=0, \quad v_{11}^{\prime}=0, \quad \theta_{11}=0 \quad \text { at } & y=1 .
\end{array}
$$

Solutions of Eqs (3.17)-(3.20) subject to Eqs (3.21) and on using Eqs (3.6)-(3.8) yield

$$
\begin{aligned}
& v_{1}(y, z)=\left[A_{5} e^{-m_{5} y}+A_{6} e^{-m_{6} y}+A_{7} e^{\pi y}+A_{8} e^{-\pi y}\right] \cos (\pi z), \\
& w_{1}(y, z)=\frac{1}{\pi}\left[A_{5} m_{5} e^{-m_{5} y}+A_{6} m_{6} e^{-m_{6} y}-A_{7} \pi e^{\pi y}+A_{8} \pi e^{-\pi y}\right] \sin (\pi z), \\
& p_{1}(y, z)=\frac{1}{\pi}\left[A_{7}(\pi-1 / K) e^{\pi y}+A_{8}(\pi+1 / K) e^{-\pi y}\right] \cos (\pi z) \\
& \theta_{1}(y, z)=\left[B_{1} e^{-\lambda_{1} y}+B_{2} e^{-\lambda_{2} y}+K_{1}\left\{B_{3} e^{-\left(m_{1}+m_{5}\right) y}+B_{4} e^{-\left(m_{1}+m_{6}\right) y}+\right.\right. \\
& \left.+B_{5} e^{\left(\pi-m_{1}\right) y}+B_{6} e^{-\left(\pi+m_{1}\right) y}\right\}+K_{2}\left\{B_{7} e^{-\left(m_{2}+m_{5}\right) y}+\right. \\
& \left.\left.+B_{8} e^{-\left(m_{2}+m_{6}\right) y}+B_{9} e^{\left(\pi-m_{2}\right) y}+B_{10} e^{-\left(\pi+m_{2}\right) y}\right\}\right] \cos (\pi z), \\
& u_{1}(y, z)=\left[A e^{-m_{5} y}+B e^{-m_{6} y}+C e^{-\lambda_{1} y}+D e^{-\lambda_{2} y}+\sum_{i=1}^{4} C_{i} e^{-\left(m_{i}+m_{5}\right) y}+\right. \\
& \left.+\sum_{i=1}^{4} D_{i} e^{-\left(m_{i}+m_{6}\right) y}+\sum_{i=1}^{4} E_{i} e^{\left(\pi-m_{i}\right) y}+\sum_{i=1}^{4} F_{i} e^{-\left(\pi+m_{i}\right) y}\right] \cos (\pi z)
\end{aligned}
$$

where

$$
\begin{aligned}
& m_{5}=\frac{1}{2}\left\{\operatorname{Re}+\sqrt{\operatorname{Re}^{2}+4\left(\pi^{2}+4 \operatorname{Re} / K\right)}\right\}, \\
& m_{5}=\frac{1}{2}\left\{\operatorname{Re}-\sqrt{\operatorname{Re}^{2}+4\left(\pi^{2}+4 \operatorname{Re} / K\right)}\right\}, \\
& \lambda_{3}=\frac{1}{2}\left\{\operatorname{RePr}+\sqrt{\operatorname{Re}^{2} \operatorname{Pr}^{2}+4\left(F \operatorname{RePr}+\pi^{2}\right)}\right\},
\end{aligned}
$$




$$
\begin{aligned}
& \lambda_{4}=\frac{1}{2}\left\{\operatorname{RePr}-\sqrt{\operatorname{Re}^{2} \operatorname{Pr}^{2}+4\left(F \operatorname{RePr}+\pi^{2}\right)}\right\}, \\
& A_{5}=\left[\pi r_{2}\left(e^{\pi}-e^{-\pi}\right)+r_{4}\left(e^{\pi}+e^{-\pi}\right)\right] / 2\left(r_{1} r_{4}-r_{2} r_{3}\right), \\
& A_{6}=-\left[\pi r_{1}\left(e^{\pi}-e^{-\pi}\right)+r_{4}\left(e^{\pi}+e^{-\pi}\right)\right] / 2\left(r_{1} r_{4}-r_{2} r_{3}\right), \\
& A_{7}=-\frac{1}{2 \pi}\left[\pi+A_{5}\left(\pi-m_{5}\right)+A_{6}\left(\pi-m_{6}\right)\right], \\
& A_{8}=-\frac{1}{2 \pi}\left[\pi+A_{5}\left(\pi+m_{5}\right)+A_{6}\left(\pi+m_{6}\right)\right], \\
& r_{1}=e^{-m_{5}}-\frac{1}{2 \pi}\left[e^{\pi}\left(\pi-m_{5}\right)+e^{-\pi}\left(\pi+m_{5}\right)\right], \\
& r_{2}=e^{-m_{6}}-\frac{1}{2 \pi}\left[e^{\pi}\left(\pi-m_{6}\right)+e^{-\pi}\left(\pi+m_{6}\right)\right], \\
& r_{3}=m_{5} e^{-m_{5}}+\frac{1}{2}\left[e^{\pi}\left(\pi-m_{5}\right)-e^{-\pi}\left(\pi+m_{5}\right)\right], \\
& r_{6} e_{6}+\frac{1}{2}\left[e^{\pi}\left(\pi-m_{6}\right)-e^{-\pi}\left(\pi+m_{6}\right)\right] .
\end{aligned}
$$

The other constants are not given here to save space. If $K \rightarrow \infty$ the results are same as Guria and Jana (2010).

\section{Results and discussion}

In order to get a physical insight into the problem the velocity field, temperature field, shear stresses and Nusselt number have been discussed for various parameters. We have plotted the non-dimensional primary velocity $u$ in Figs 2-5 for different values of the Grashof number, radiation parameter, permeability parameter and Prandtl number for $\operatorname{Re}=5.0, \varepsilon=0.05, z=0.0$. It is observed from Fig. 2 that greater cooling of the surface (an increase in Gr)results in an increase in the velocity. It is due to the fact that the values of the thermal Grashof number has the tendency to increase the thermal buoyancy effect. This gives rise to an increase in the induced flow. It is seen from Figs 3 and 4 that the primary velocity decreases with an increase in the radiation parameter as well as the Prandtl number for cooling of the plate $(\mathrm{Gr}>0)$. The reverse effect is observed in case of heating of the plate $(\mathrm{Gr}<0)$. It is found from Fig. 5 that the primary velocity increases with an increase in the permeability parameter for cooling of the plate. The reverse effect is observed in case of heating of the plate. The effect of the permeability parameter $K$ on secondary velocity is shown in Fig. 6 for $\operatorname{Re}=5.0, \varepsilon=0.05, z=0.5$. It is found that the magnitude of the secondary velocity decreases near the plate $y=0$ and increases near the plate $y=1$ with an increase in the permeability parameter. 


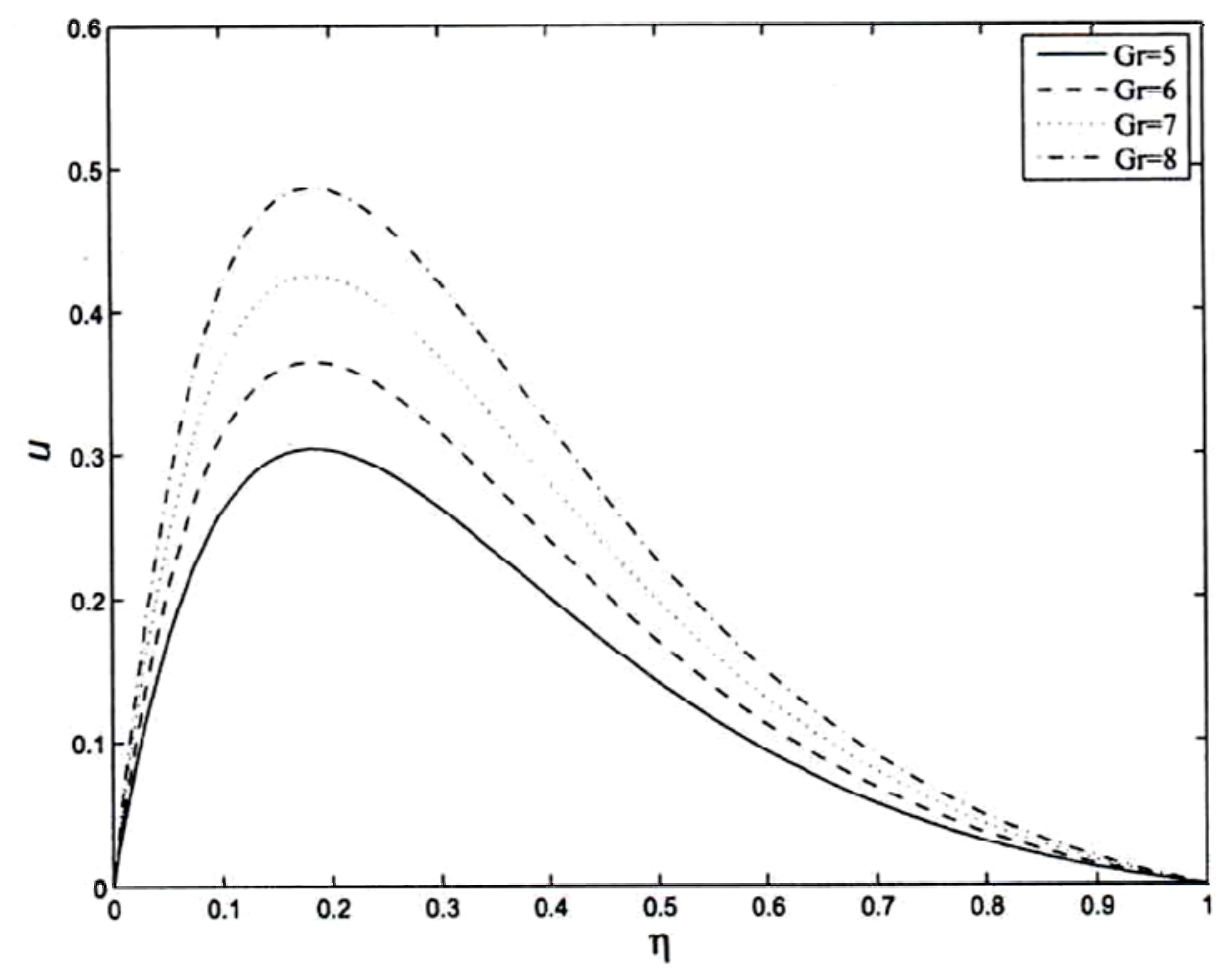

Fig.2. Primary velocity $u$ for $\operatorname{Re}=5.0, \operatorname{Pr}=0.71, K=0.5, \varepsilon=0.05, z=0.0$.

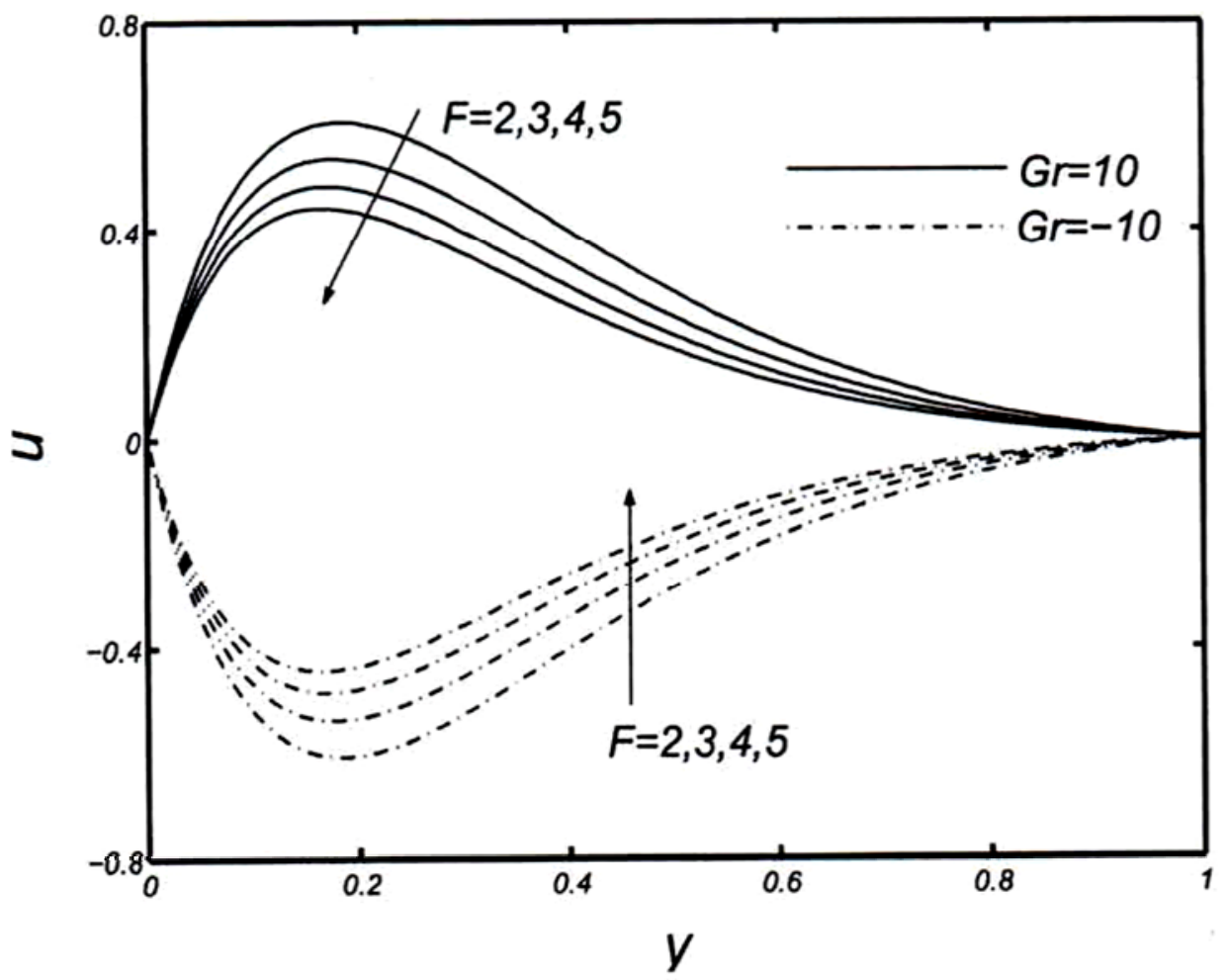

Fig.3. Primary velocity $u$ for $\operatorname{Re}=5.0, \operatorname{Pr}=0.71, K=0.5, \varepsilon=0.05, z=0.0$. 


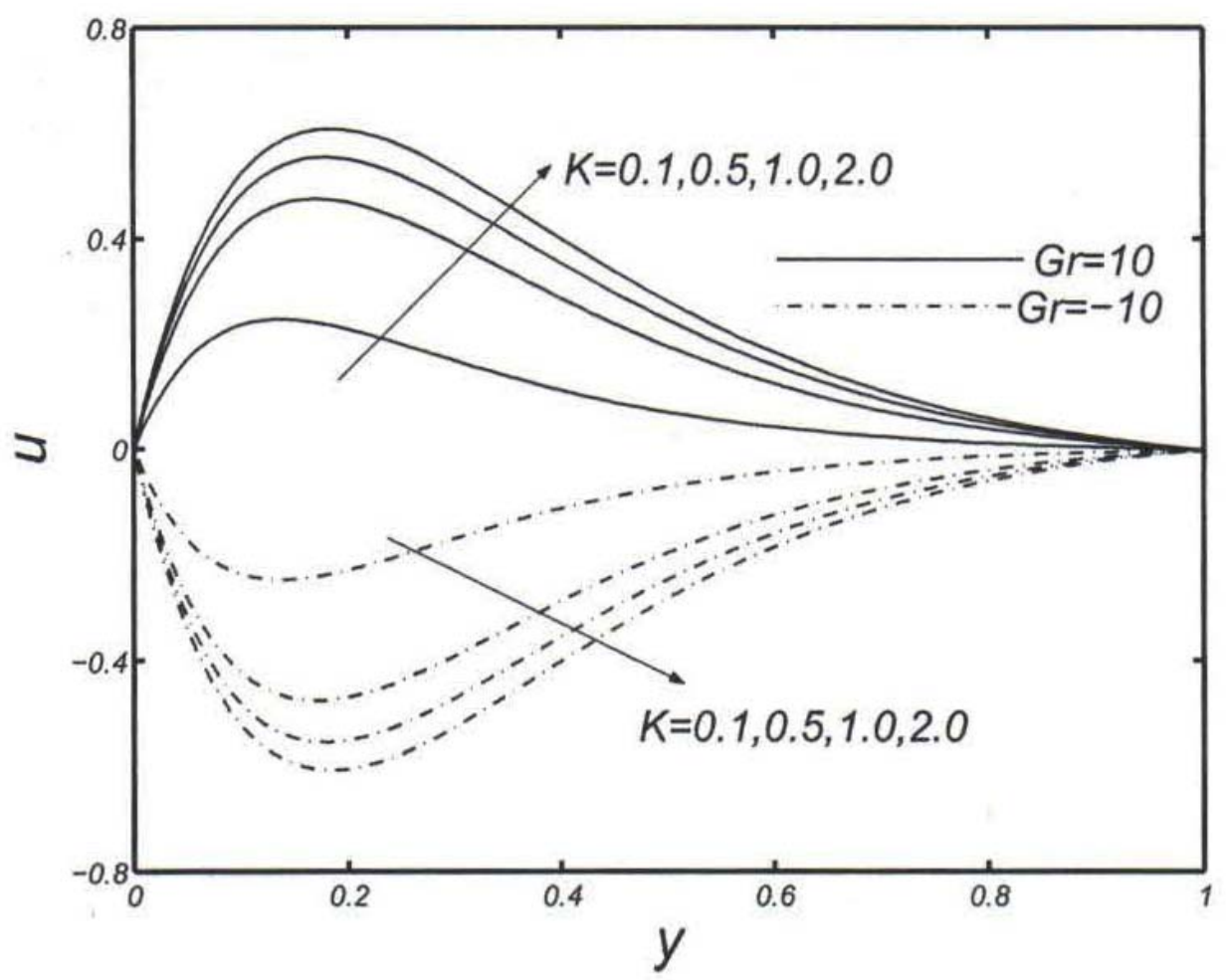

Fig.4. Primary velocity $u$ for $\operatorname{Re}=5.0, \operatorname{Pr}=0.71, F=2.0, \varepsilon=0.05, z=0.0$.

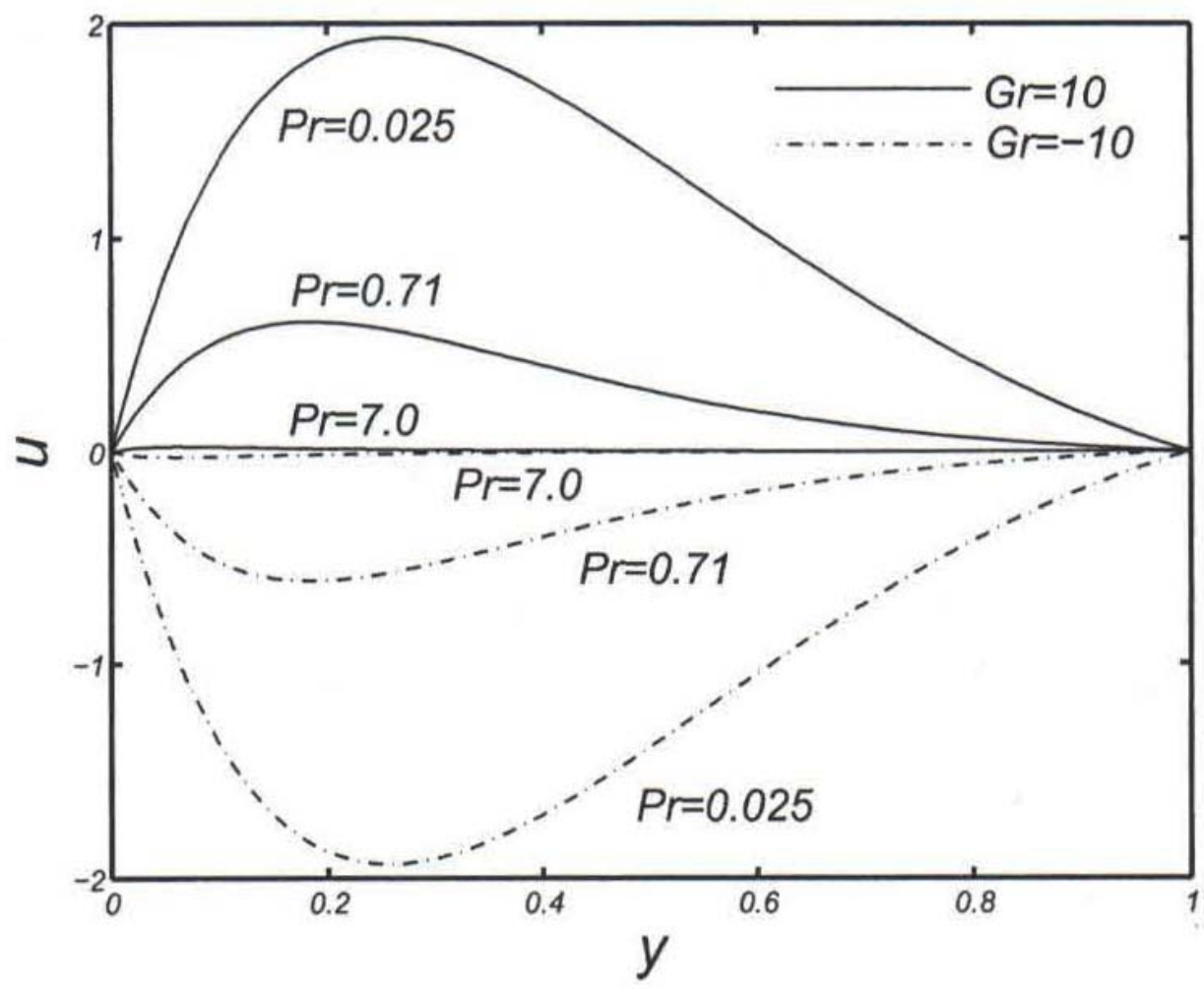

Fig.5. Primary velocity $u$ for $\mathrm{Re}=5.0, \mathrm{~K}=0.5, F=2.0, \varepsilon=0.05, z=0.0$. 


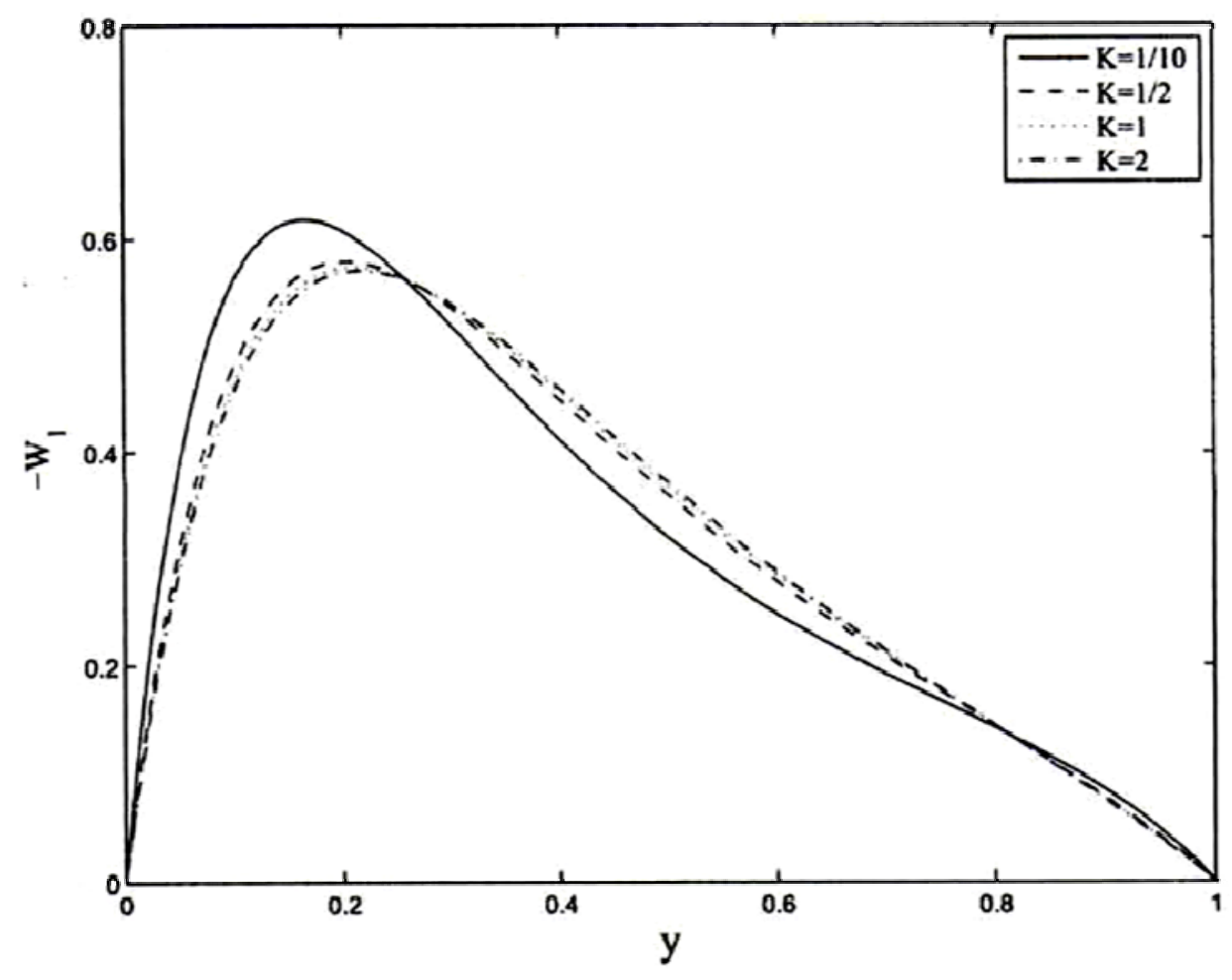

Fig.6. Secondary velocity $-W_{1}$ for $\mathrm{Re}=5.0, \varepsilon=0.05, z=0.5$.

Knowing the velocity field it is interesting to know the shear stress at the plate. The shear stress at the plate $y^{*}=0$ due to the primary flow is given by

$$
\tau_{x}^{*}=\mu\left(\frac{\partial u^{*}}{\partial y^{*}}\right)_{y^{*}=0}=\frac{\mu V_{0}}{d}\left(\frac{\partial u}{\partial y}\right)_{y=0}
$$

In a non-dimensional form the shear stress at the plate $y=0$ can be written as

$$
\begin{aligned}
& \tau_{x}=\frac{\tau_{x}^{*} d}{\mu V_{0}}=\left(\frac{\partial u}{\partial y}\right)_{y=0} \\
& =u_{0}^{\prime}(0)+\varepsilon u_{1}^{\prime}(0) \\
& =-\sum_{i=1}^{4} A_{i} m_{i}+\varepsilon\left[-A m_{5}-B m_{6}-C \lambda_{1}-D \lambda_{2}-\sum_{i=1}^{4} C_{i}\left(m_{i}+m_{5}\right)+\right. \\
& \left.-\sum_{i=1}^{4} D_{i}\left(m_{i}+m_{6}\right)+\sum_{i=1}^{4} E_{i}\left(\pi-m_{i}\right)-\sum_{i=1}^{4} E_{i}\left(\pi-m_{i}\right)\right] \cos (\pi z) .
\end{aligned}
$$

The shear stress due to the primary flow in terms of $\tau_{x}$ is shown in Tab.1 for different values of the Reynolds number and radiation parameter for cooling of the plate. It is seen that $\tau_{x}$ increases with an increase in the Reynolds number but it decreases with an increase in the radiation parameter for cooling of the plate. 
Table 1. Shear stress component due to the primary flow for $K=2, \mathrm{Gr}=10.0, \operatorname{Pr}=0.71, \varepsilon=0.05, z=0.0$.

\begin{tabular}{|c|c|c|c|c|}
\hline & \multicolumn{4}{|c|}{$\tau_{x}$} \\
\hline $\operatorname{Re}$ & $F=2.0$ & $F=3.0$ & $F=4.0$ & $F=5.0$ \\
\hline 2 & 5.320 & 5.003 & 4.737 & 4.510 \\
\hline 4 & 8.147 & 7.522 & 7.030 & 6.630 \\
\hline 6 & 9.545 & 8.813 & 8.244 & 7.785 \\
\hline 8 & 10.796 & 9.576 & 9.000 & 8.531 \\
\hline
\end{tabular}

The effect of the permeability parameter on $\tau_{x}$ is shown in Tab.2 for cooling of the plate. It is found that $\tau_{x}$ increases with an increase in the permeability parameter.

Table 2. Shear stress component due to primary flow for $\mathrm{Gr}=10.0, \operatorname{Pr}=0.71, \varepsilon=0.05, z=0.0$.

\begin{tabular}{|c|c|c|c|c|}
\hline & \multicolumn{4}{|c|}{$\tau_{x}$} \\
\hline $\operatorname{Re}$ & $K=1 / 10$ & $K=1 / 2$ & $K=1$ & $K=2$ \\
\hline 2 & 3.155 & 4.661 & 5.070 & 5.320 \\
\hline 4 & 4.517 & 6.909 & 7.655 & 8.147 \\
\hline 6 & 5.432 & 8.157 & 8.993 & 9.545 \\
\hline 8 & 6.130 & 8.962 & 9.782 & 10.311 \\
\hline
\end{tabular}

The shear stress due to the secondary flow can be expressed as

$$
\tau_{z}^{*}=\mu\left(\frac{\partial w^{*}}{\partial y^{*}}\right)_{y^{*}=0}=\frac{\mu V_{0}}{d}\left(\frac{\partial w}{\partial y}\right)_{y=0}
$$

In a non-dimensional form the shear stress due to the secondary flow at the plate $y=0$ can be written as

$$
\begin{aligned}
& \tau_{z}=\frac{\tau_{x}^{*} d}{\mu V_{0}}=\left(\frac{\partial w}{\partial y}\right)_{y=0} \\
& =w_{0}^{\prime}(0)+\varepsilon w_{1}^{\prime}(0) \\
& =-\frac{\varepsilon}{\pi}\left[A_{5} m_{5}^{2}+A_{6} m_{6}^{2}+A_{7} \pi^{2}+A_{8} \pi^{2}\right] \sin (\pi z) .
\end{aligned}
$$

The shear stress due to the secondary flow in terms of $\tau_{z}$ is given in Tab.3 for several values of the Reynolds number and permeability parameter for $\varepsilon=0.05, z=0.5$. It is observed that the magnitude of $\tau_{z}$ increases with an increase in the Reynolds number whereas it decreases with an increase in the permeability parameter.

Table 3. Shear stress component due to the secondary flow for $\varepsilon=0.05, z=0.5$.

\begin{tabular}{|c|c|c|c|c|c|}
\hline $\operatorname{Re}$ & & & $-\tau_{z}$ & & \\
\hline & $K=1 / 10$ & $K=1 / 2$ & $K=1$ & $K=2$ & \\
\hline 2 & .341 & .265 & .254 & .248 & \\
\hline 4 & .478 & .356 & .337 & .327 & \\
\hline 6 & .605 & .452 & .428 & .416 & \\
\hline 8 & .726 & .550 & .524 & .510 & \\
\hline
\end{tabular}


The temperature $\theta$ is plotted for different values of the radiation parameter and Prandtl number in Figs 7 and 8 for $\operatorname{Re}=5.0, \mathrm{Gr}=5.0, \varepsilon=0.05, z=0.0$ for cooling of the plate. It is found that the temperature $\theta$ decreases with an increase in the radiation parameter as well as the Prandtl number. This is due to fact that thermal conductivity of the fluid decreases with increasing Pr, resulting in a decrease of thermal boundary layer thickness.

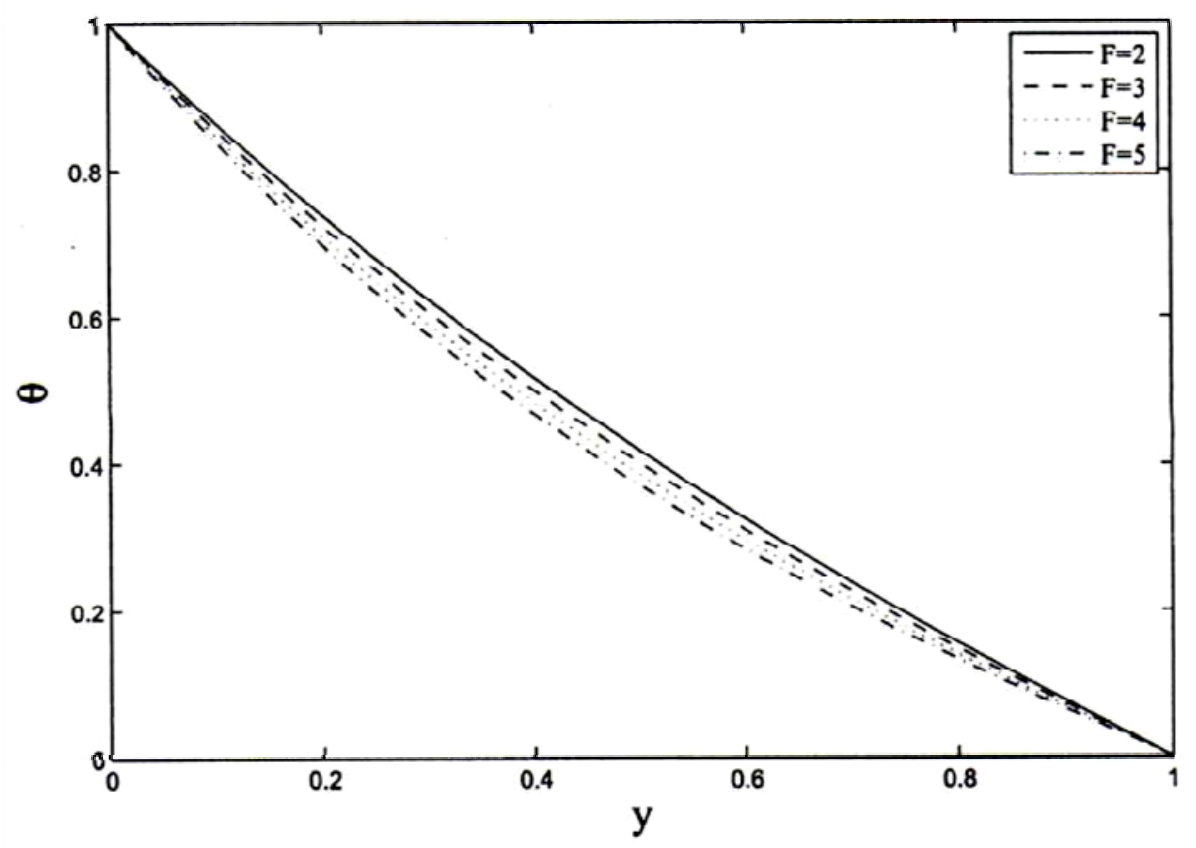

Fig.7. Temperature profile $\theta$ for $\mathrm{Gr}=5.0, \operatorname{Re}=5.0, \operatorname{Pr}=0.71, K=0.5, \varepsilon=0.05, z=0.0$.

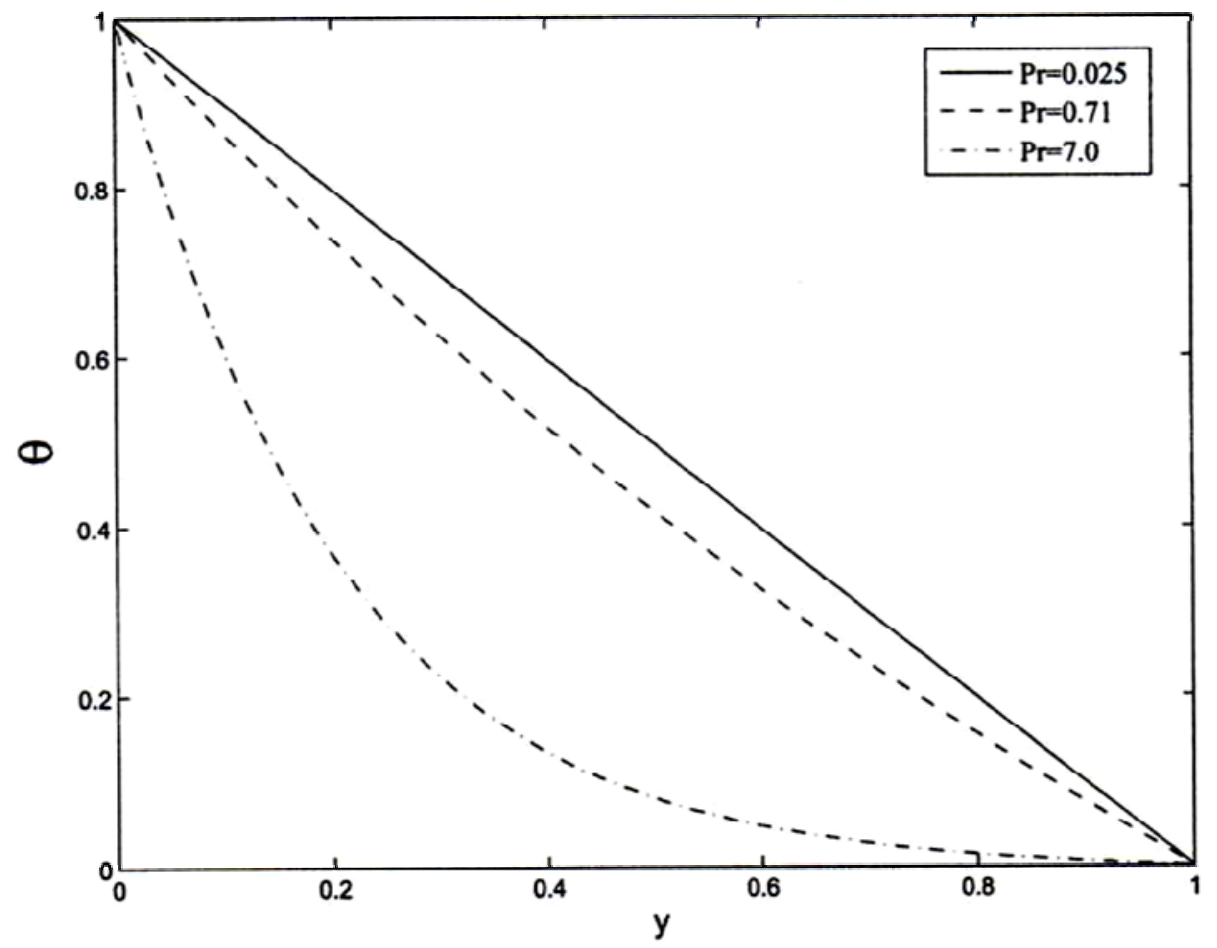

Fig.8. Temperature profile $\theta$ for $\mathrm{Gr}=5.0, \mathrm{Re}=5.0, K=0.5, F=2, \operatorname{Pr}=0.71, \varepsilon=0.05, z=0.0$. 
The Nusselt number is the great measure of heat transfer from the plate to the fluid flowing up between the plates due to its practical importance. The Nusselt number depends on the rate of heat transfer from the plate to the fluid. The heat transfer coefficient from the plate to the fluid may be calculated as

$$
q=-k\left(\frac{\partial T^{*}}{\partial y^{*}}\right)_{y^{*}=0}=-\frac{k\left(T_{w}-T_{0}\right)}{d}\left(\frac{\partial \theta}{\partial y}\right)_{y=0}
$$

In a non-dimensional form the heat transfer coefficient at the plate $y=0$ is given by

$$
\begin{aligned}
& \mathrm{Nu}=\frac{q d}{k\left(T_{w}-T_{0}\right)}=-\left(\frac{\partial \theta}{\partial y}\right)_{y=0}=-\theta_{0}^{\prime}(0)-\varepsilon \theta_{1}^{\prime}(0), \\
& =\frac{m_{2} e^{-m_{1}}-m_{1} e^{-m_{2}}}{\left(e^{-m_{2}}-e-m_{1}\right)}-\left[B_{1} \lambda_{1}+B_{2} \lambda_{2}+K_{1} B_{4}\left(m_{1}+m_{6}\right)-K_{1} B_{5}\left(\pi-m_{1}\right)+\right. \\
& \left.+K_{1} B_{6}\left(\pi+m_{1}\right)+K_{2} B_{7}\left(m_{2}+m_{5}\right)+K_{2} B_{8}\left(m_{2}+m_{6}\right)+K_{2} B_{10}\left(\pi+m_{2}\right)\right] \cos (\pi z) .
\end{aligned}
$$

Table 4. Nusselt number for $\mathrm{Gr}=10.0, \operatorname{Pr}=0.71, \varepsilon=0.05, z=0.0$.

\begin{tabular}{|c|c|c|c|c|}
\hline & \multicolumn{4}{|c|}{$\mathrm{Nu}$} \\
\hline $\mathrm{Re}$ & $F=2$ & $F=3$ & $F=4$ & $F=5$ \\
\hline 2 & 2.663 & 2.978 & 3.261 & 3.520 \\
\hline 4 & 4.288 & 4.749 & 5.156 & 5.524 \\
\hline 6 & 5.873 & 6.418 & 6.901 & 7.338 \\
\hline 8 & 7.431 & 8.032 & 8.568 & 9.057 \\
\hline
\end{tabular}

Table 5. Nusselt number for $\mathrm{Gr}=10.0, \operatorname{Pr}=0.71, \varepsilon=0.05, z=0.0$.

\begin{tabular}{|c|c|c|c|c|}
\hline & \multicolumn{4}{|c|}{$\mathrm{Nu}$} \\
\hline $\mathrm{Re}$ & $K=1 / 10$ & $K=1 / 2$ & $K=1$ & $K=2$ \\
\hline 2 & 2.662 & 2.663 & 2.663 & 2.663 \\
\hline 4 & 4.286 & 4.287 & 4.288 & 4.288 \\
\hline 6 & 5.870 & 5.873 & 5.873 & 5.873 \\
\hline 8 & 7.427 & 7.430 & 7.431 & 7.431 \\
\hline
\end{tabular}

The rate of heat transfer in terms of the Nusselt number for different values of the radiation parameter, permeability parameter and Reynolds number and for $\mathrm{Gr}=5.0, \varepsilon=0.05, z=0.0$ are given in Tabs 4 and 5 for cooling of the plate. The Nusseltl number increases with an increase in the Reynolds number as well as the radiation parameter. Also it increases with an increase in the permeability parameter but the effect is very small.

\section{Conclusion}

The steady flow of a viscous incompressible fluid passing through a vertical channel through a porous medium has been studied in the presence of radiation. It is found that the primary velocity decreases 
with an increase in the radiation parameter as well as the Prandtl number for cooling of the plate. It is also found that with an increase in the permeability parameter the primary velocity increases for cooling of the plate. On the other hand, the magnitude of the secondary velocity decreases near the left plate and increases near the right plate with an increase in the permeability parameter. The shear stress due to the primary flow increases either in the Reynolds number or permeability parameter but it decreases with increase in radiation parameter. The magnitude of the shear stress due to the secondary flow increases with an increase in the Reynolds number but it decreases with an increase in the Reynolds number but it decreases with an increase in the permeability parameter. It is observed that the temperature profile decreases with an increase in either the radiation parameter or the Prandtl number for cooling of the plate. The rate of heat transfer in terms of the Prandtl number, increases with an increase in either the Reynolds number or the radiation parameter.

\section{Acknowledgement}

The author thanks the UGC of India for financial support for carrying out the research via a Minor Research Project in the year 2012-13 of No. F. PSW-86/12-13(ERO).

\section{Nomenclature}

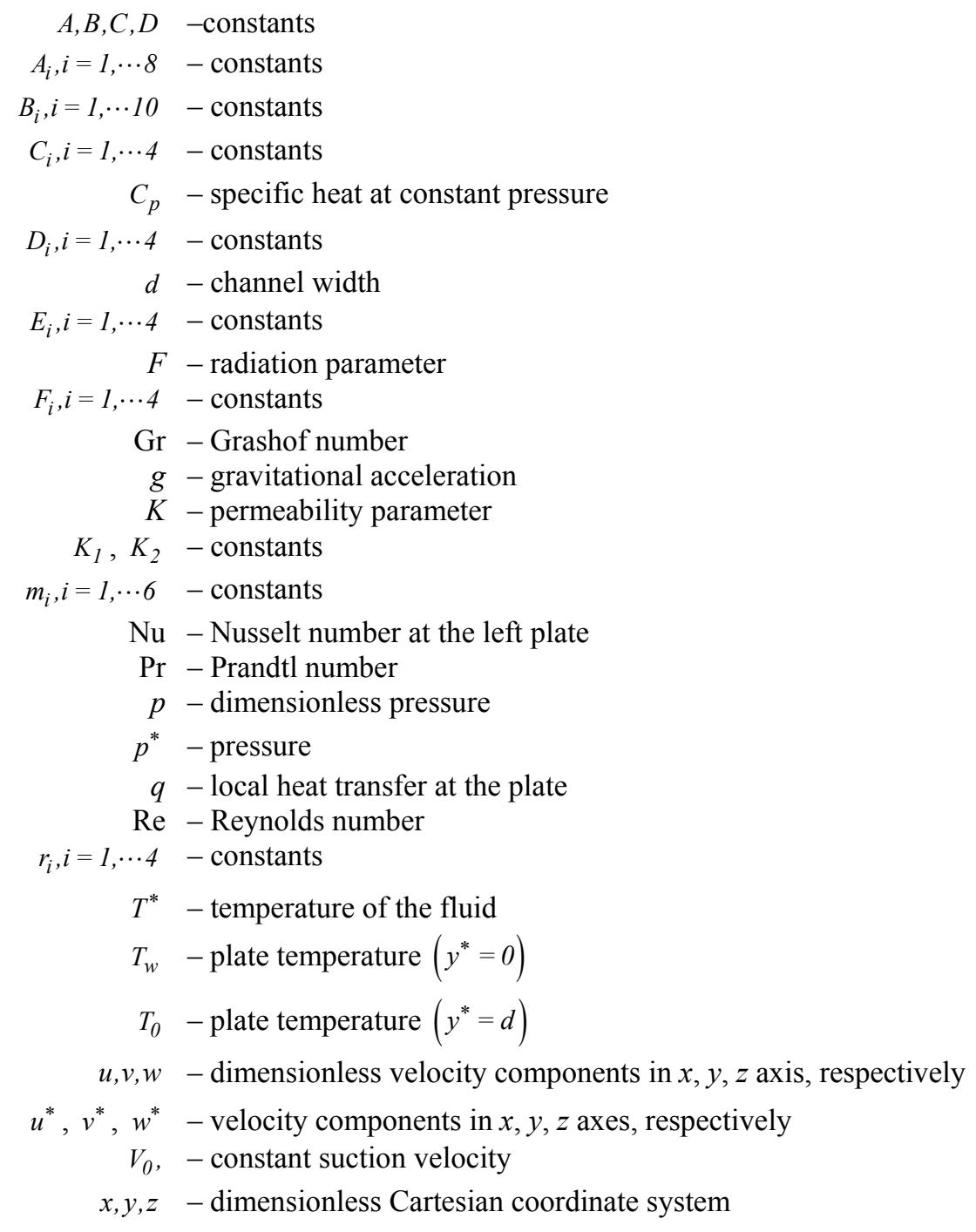




$$
\begin{aligned}
x^{*}, y^{*}, z^{*} & - \text { Cartesian coordinate system } \\
\beta & - \text { coefficient of thermal expansion } \\
\varepsilon & - \text { amplitude of the suction velocity } \\
\theta & - \text { non-dimensional temperature } \\
\lambda_{i}, i=1 \cdots 4 & - \text { constants } \\
\mu & - \text { viscosity } \\
\nu & - \text { kinematic viscosity } \\
\rho & - \text { density } \\
\tau_{x} & - \text { shear stress due to primary flow } \\
\tau_{z} & - \text { shear stress due to secondary flow }
\end{aligned}
$$

\section{References}

Ahmed S. and Ahmed N. (2004): Two dimensional MHD oscillatory flow along a uniformly moving infinite vertical porous plate bounded by a porous medium. - Indian J. Pure Appl. Math., vol.35, No.12, pp.1309-13.

Guria M., Ghara N. and Jana R.N. (2010): Radiation effect on three dimensional vertical channel flow. - Int. J. Applied Mechanics and Engineering, vol.15, No.4, pp.1065-1081.

Guria M., Ghara N. and Jana R.N. (2011): Radiation effect on the three-dimensional MHD flow past a vertical porous plate. - Journal of Physical Sciences, vol.15, pp.161-170.

Pathak G., Maheswari C.H. and Gupta S.P. (2006): Effect of radiation on unsteady free convection flow bounded by an oscillating plate with variable wall temperature. - Int. J. Applied Mechanics and Engineering, vol.11, No.2, pp.371382.

Raptis A. (1983): Unsteady free convective flow through a porous medium. - Int. J. Engng. Sci., vol.21, pp.345-48.

Raptis A. (1998): Radiation and free convection flow through a porous medium. - Int. Comm. Heat Mass Transfer, vol.25, No.2, pp.289-295.

Raptis A. and Perdikis C.P. (1985): Oscillatory flow through a porous medium in the presence of free convective flow.Int. J. Engng. Sci., vol.23, pp.51-55.

Seddeek MA. (2000): The effect of variable viscosity on hydromagnetic flow and heat transfer past a continuously moving porous boundary with radiation. - Int. Comm. Heat Mass Transfer, vol.27, No.7, pp.1037-1046.

Sharma B.K., Agarwal M. and Chaudhary R.C. (2007): Radiation effect on temperature distribution in three dimensional Couette flow with suction injection. - Applied Mathematics Mechanics, vol.28, No.3, pp.309-316.

Singh P., Mishra J.K. and Narayanan K.A. (1988): Three dimensional convective flow through a porous medium with variable suction velocity. - Indian J. Pure Appl. Math. vol.19, pp.1130.

Takhar H.S., Gorla R.S.R. and Soundalgekar V.M. (1996): Radiation effects on MHD free convection flow of a radiating gas past a semi-infinite vertical plate. - Int. J. Numer. Meth. Heat Fluid Flow, vol.6, No.2, pp.77-83.

Varshney C.L. (1979): Oscillatory two dimensional flow through a porous medium by a porous plate. - Indian J. Pure Appl. Math., vol.10, pp.1558-1564.

Received: June 2, 2014

Revised: September 12, 2015 